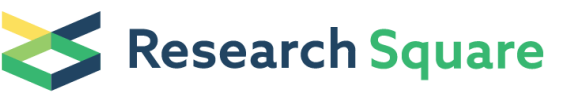

\section{Epigenetic silencing and tumor suppressor gene of HAND2 by targeting ERK signaling in colorectal cancer}

\section{Zixu Yuan ( $\nabla$ yuanzx@mail2.sysu.edu.cn )}

Sun Yat-sen University Sixth Affiliated Hospital https://orcid.org/0000-0001-8249-3352

\section{Xihu Yu}

Sun Yat-sen University Sixth Affiliated Hospital

Jian Cai

Sun Yat-sen University Sixth Affiliated Hospital

\section{Daici Chen}

Sun Yat-sen University Sixth Affiliated Hospital

\section{Xiaoxia Liu}

Sun Yat-sen University Sixth Affiliated Hospital

\section{Zhijie Wu}

Sun Yat-sen University Sixth Affiliated Hospital

\section{Wenle Chen}

Sun Yat-sen University Sixth Affiliated Hospital

William M. Grady

Sun Yat-sen University Sixth Affiliated Hospital

Hui Wang

Sun Yat-sen University Sixth Affiliated Hospital

\section{Chun Chen}

Sun Yat-sen University Sixth Affiliated Hospital

\section{Research}

Keywords: HAND2, epigenetics, colorectal cancer, ERK signaling

Posted Date: October 18th, 2021

DOI: https://doi.org/10.21203/rs.3.rs-945202/v1

License: (c) (1) This work is licensed under a Creative Commons Attribution 4.0 International License. Read Full License 


\section{Abstract \\ Background}

The screening biomarkers for early detection of colorectal cancer (CRC) is lacking. The aim is to identify epigenetic silenced genes and clarify its roles and underlying mechanism in CRC. We conducted integrative analyses of epigenome-wide Human Methylation 450K arrays and transcriptome to screen out candidate epigenetic driver genes with transcription silencing. Methylated silencing HAND2 were identified and verified in large CRC cohort. The mechanism of HAND2 expression by promoter inhibition were clarified both in vitro and vivo assays. Cell biofunctional roles of HAND2 methylation was investigated in CRC cells. HAND2 reconstitution were constructed by lentivirus plasmid and tumor xenograft model of HAND2 were built subcutaneously. Genomic mRNA analysis by RNA-sequencing and subsequent GSEA analysis were performed to identify potential target of HAND2 and qPCR/WB was conducted to identify the results.

\section{Results}

We firstly reported high frequency of HAND2 methylation in promoter in CRC and hypermethylation was negatively correlated with expression silencing and leaded to poor survival in several CRC cohort patients. 5-Aza treatment to demethylated HAND2 could revert its expression in CRC cells. Functionally, HAND2 reconstitution can inhibit cell proliferation, invasion and migration in vitro. In tumor xenograft, HAND2 reconstruction significantly repressed tumor growth when compared to control vector. Thousands of aberrant expressed genes were observed in the heatmap of RNA-sequencing data. HAND2 reconstitution could bind to ERK and reduce its phosphorylation by CoIP assay. These above results showed HAND2 reconstitution perturbed the activation of MAPK/ERK signaling by reduction of ERK phosphorylation.

\section{Conclusions}

HAND2 is one tumor suppressor by targeting ERK signaling and one potential epigenetic driver gene in CRC.

\section{Background}

Colorectal cancer (CRC) is the third most common cancer and ranks the third of cancer death worldwide

${ }^{1}$. Aberrant epigenetic alterations are common hallmarks during tumorigenesis, including hypermethylation in CpG-rich sequences, also known as CpG islands in gene promotor region, and hypomethylation in global DNA region ${ }^{2}$. Dysregulated DNA methylation is involved in carcinogenesis, progression and maintenance in many types of cancers. DNA methylation in promoter regions can lead to silencing of mRNA transcription and decreased protein expression in many genes ${ }^{3,4}$. In addition, promoter hypermethylation in a group of epigenetic driver genes are shown to be key epigenetic early 
events associated with cancer cell survival. Cancer cells become addicted and susceptible to aberrant DNA methylation ${ }^{3}$. Identification of these driver genes that occurred at the beginning of carcinogenesis will help to develop more epigenetic targeted therapies. FDA has approved a few methylated biomarkers for CRC screening test, such as promoter methylation of BMP3, NDRG4 in Cologuard kit of stool test ${ }^{5}$, and circulating Septin 9 in Epi proColon kit in the blood. However, the sensitivity and specificity of these biomarkers for early detection of CRC are still limited, it is pivotal to find more epigenetic driver genes for potential biomarkers. Artificial intelligence (Al) is also important to diagnose CRC in our recent study published in Annals of Surgery 6 .

Advanced epigenomic technology is now widely used in epigenome-wide analyses of cancer methylome. Thousands of aberrant DNA methylated genes in cancer are found and thus make it possible to identify cancer specific methylation and driver genes that play key roles in early carcinogenesis and cancer cell survival. Methylation levels can also be used to classify cancer subtypes and predict cancer outcomes ${ }^{7}$. However, distinguish these driver genes through thousands of methylated $\mathrm{CpGs}$ need tremendous efforts worldwide. Thus, large volumes of high throughput methylome profiles in cancer epigenome are submitted to one public database called The Cancer Genome Atlas (TCGA). Meanwhile, newly developed genome-wide mRNA sequencing technology produces big data of gene transcriptions in human cancers. It is easier to screen out potential driver genes through the integrative analysis of epigenometranscriptome big datasets in CRC.

In this study, to identify epigenetic driver genes that are hypermethylated in the promoter along with transcription silencing, we firstly conducted integrative analyses of epigenome-wide arrays including Human Methylation 27K/450K (HM27K/450K) beadchip arrays and transcriptome including RNA sequencing datasets. About 600 patients with colon cancers from TCGA database and our own unpublished datasets are included. We have identified many candidate genes through integrate bioinformatic analysis. In this study, we focus on one most promising driver gene, heart and neural crest derivatives expressed 2 (HAND2), a transcriptional factor. Epigenetic silenced TF often regulates functional genes by promoter binding and influences downstream signaling in cancer cell survival.

Recently, Jones et al. establishes critical role of epigenetic silenced HAND2 in endometrial cancer development ${ }^{8}$. Hypermethylated HAND2 and silencing is early molecular event in carcinogenesis, and provide as one biomarker for early detection of endometrial cancer. However, little is known about the role of HAND2 in CRC and other cancer types. In this study, we will investigate the functional roles and molecular mechanism of HAND2 DNA methylation and epigenetic silencing in CRC. The downstream signaling of HAND2 will also be clarified by high throughput RNA sequencing and validated both in vitro and vivo assays. Novel epigenetic biomarkers for early detection of CRC and targeted therapies are potential to be developed for precision medicine.

\section{Results}




\section{Aberrant methylation of HAND2 in the promoter is frequent in CRC}

Firstly, we conducted epigenome-transcriptome-interactome integrated bioinformatic analyses of TCGA CRC dataset and some of our own CRC patients in Seattle lab, Washington. We have found 149 aberrant methylated $\mathrm{CpG}$ locus. Our focus was on these $\mathrm{CpG}$ locus firstly reported in $\mathrm{CRC}$, located in the gene promoter region, and acted potential oncological roles. Finally, we identified one CpG cite (cg02774439) in the promoter of HAND2, which is the only methylated CpG cite of HAND2 gene.

In a Chinese cohort of CRC patients, HAND2 methylation was greatly increased in CRC tissue than paired normal tissues (tumor: $2.73 \% \pm 3.43 \%$; normal: $0.31 \% \pm 0.40 \% ; n=74$ pairs, $P<0.001$ ) by qMSP (Figure $1 \mathrm{~A}$ ). In addition, hypermethylation of HAND2 was found in 52/74 (70.3\%) of CRC, which was defined as $>60 \%$ methylation by referring to endogenous control ALUC4. The methylation levels of HAND2 were at least 10 times more than paired normal tissues in 64/74 (86.5\%) of tumors. In addition, the frequency of high methylation of HAND2 promoter with $\beta$ value $>0.5$ was found in $64 \%(142 / 223)$ of patients in TCGA cohort. The percent of intermediate methylation with a $0.3<\beta$ value $<0.5$ was $24 \%(53 / 223)$.

\section{Aberrant methylation of HAND2 silenced gene expression and predicted poor prognosis in CRC}

To clarify the role of aberrant methylation on mRNA expression, HAND2 mRNA expression was found to be decreased dramatically in CRC tissues than paired normal tissues $(n=70$ pairs, $P<0.001)$ in Chinese cohort 1 (Figure 1B). To verify HAND2 mRNA expression in CRC, another Chinese CRC cohort 2 was measured, and HAND2 expression decreased greatly were observed in CRC tissues $(0.958 \pm 1.474)$ than paired normal tissues $(1.836 \pm 1.583)(\mathrm{n}=86$ pairs, $\mathrm{P}<0.001)$ in (Supplement Figure $\mathrm{S1})$.

To investigate the correlation between HAND2 methylation and mRNA expression, HAND2 expression was found to be negatively correlated with its methylation ( $n=61$ pairs, $R^{2}=0.217, P<0.001$ ) (Figure 1C). Furthermore, we verified this correlation in another cohort from TCGA COAD dataset. As expected, HAND2 expression was found to be negatively correlated with its methylation in TCGA cohort ( $n=247$ pairs, $\mathrm{R}^{2}=0.155, \mathrm{P}<0.001$ ) (Supplement Figure S2).

To investigate the prognostic role of hypermethylated HAND2, we classified CRC patients into two groups with high or low methylation levels of HAND2 in Chinese cohort 1. The cutoff value of overall survival (OS) was decided by ROC curve. Patients with high methylation of HAND2 predicted decreased OS than patients with low HAND2 methylation ( $P=0.035$ ) (Figure 1D).

\section{HAND 2 methylation repressed expression in CRC cell lines}

To further investigate the effect of HAND2 methylation on expression in CRC cells, we found hypermethylated HAND2 was widely increased in almost all of CRC cell lines (Figure 2A). In addition, obvious decreased HAND2 expression was observed in a panel of CRC cell lines, when comparing to 
normal colon cells CCD18co, NCM 460 and normal cells 293T (Figure 2B). We then conducted IHC analysis and found decreased HAND2 protein expression in CRC tissue, when comparing to normal colon mucosa tissue (Figure 2C-D). Interesting, we found HAND2 expression was mainly located in the colon stroma cells (Figure 2C).

In order to determine the mechanism for loss of HAND2 expression, we found 5-Aza treatment to demethylated HAND2 and found induced HAND2 expression in DLD1 (Figure 2E). Therefore, we concluded that the methylation of HAND2 can repress HAND2 expression.

\section{Methylated HAND2 promoted cell proliferation, invasion and migration}

To investigate the effect of HAND2 on CRC, we constructed HAND2 overexpressed vector and transfected into RKO and DLD1 cells. In these cell lines, HAND2 protein expression by western blot was greatly recovered when compared to the transfected cells of control vector, which indicated the successful construction of HAND2 expressed cells (Figure 3A).

Furthermore, we determined the cellular functional roles of HAND2 in CRC. Cell proliferation was greatly decreased when HAND2 was reconstituted in both DLD1 and RKO cells by RTCA assays (Figure 3B). Cell invasion and migration capacity were also repressed by HAND2 reconstitution in DLD1 cells (Figure 3C). However, no significant effects of cell cycle were shown when HAND2 expression was rebuilt (Supplement Figure S3).

\section{HAND2 suppressed tumor growth in vivo}

We also performed studies in vivo on tumor growth and xenograft formation in constructed stable cell lines to assess the suppressor role of HAND2 in CRC. HAND2 stable cells were injected into nude mice subcutaneously. HAND2 reconstruction significantly repressed tumor growth when compared to xenografts that contained a control vector (Figure 4A-D). 24 days after the injection of CRC cells, mice were sacrificed and the tumors were resected and measured. Both the weight and volume of HAND2expressing tumors were greatly decreased compared to control xenografts (Figure 4A-D). These results indicated HAND2 acted as a tumor suppressor in CRC.

\section{HAND2 reconstruction perturbed the activation of MAPK/ERK signaling}

As shown in above experiments in vitro and vivo, HAND2 played the suppressing role in CRC. We then explore the downstream signaling pathway that were affected by HAND2. We conducted whole genomic mRNA analysis by high throughput RNA-seq in DLD1 cells with HAND2 reconstruction compared to cells with the control vector. Thousands of genes were increased or decreased in the heatmap analysis (Figure 5A). Then we conducted GSEA analysis to determine these affected pathways. Both PTEN pathway, and KRAS pathway were upregulated, while PDGF-ERK pathway was downregulated (Figure 5B-C). In order to 
confirm the RNA-seq data, we conducted western blot in cells with HAND2 reconstruction and control vector. In both DLD1 and RKO cells, decreased p-ERK levels in HAND2 overexpressed cells were observed compared to control. However, no significant changes of ERK, PTEN, RAS and EGFR levels were shown (Figure 5D). In addition, we have also conducted ColP assay in HAND2 reconstruction DLD1 cells and wiSld-type HCT8 cells. As expected, we observed HAND2 could directly bind to ERK both in DLD1 cells and HCT8 cells (Figure 5E), which indicated HAND2 reconstitution could bind to ERK and reduce its phosphorylation for ERK inactivation to inhibit tumor growth. In addition, loss of PTEN expression in HAND2 reconstitution cells was shown in mRNA level by qPCR (Supplement Figure S4), but not observed in protein level by western blot (Figure 5D). These above results indicated the reconstruction of HAND2 perturbed the activation of MAPK/ERK signaling by reduction of ERK phosphorylation.

\section{Discussion}

The average human genome contains 1000-3000 aberrant methylation genes in CRC $^{4}$. High-throughput epigenome-wide arrays identify a large number of de novo methylation in gene promoter regions in CRC patients in our previous study ${ }^{9}$. However, it is a challenging task to discriminate epigenetic driver genes, because many aberrant methylation genes obtain no effect on mRNA expression and tumor information like "passengers" ${ }^{10}$. In this study, we firstly identified HAND2 through epigenome-transcriptome integrated bioinformatic analyses. High frequency of HAND2 cancer-specific methylation and silenced expression and poor survival are shown in CRC. HAND2 reconstitution can inhibit cell proliferation, invasion and migration in vitro, and also suppress CRC growth in tumor xenograft. Through RNAsequencing assays and subsequent GSEA analysis, we found HAND2 reconstitution perturbed the activation of MAPK/ERK signaling by reduction of ERK phosphorylation. Therefore, HAND2 exhibits classical features of tumor suppressor gene in CRC.

HAND2 belongs to the basic helix-loop-helix family of transcription factors. HAND2 involves mainly biological roles such as development in ventricular chambers, cardiac morphogenesis, angiogenesis, and enteric nerve system ${ }^{11}$, and plays anti-proliferation role in uterine epithelium ${ }^{12-14}$. In this study, we firstly identify methylated HAND2 with silencing expression and reveals functional roles of HAND2 in CRC through two independent Chinese cohorts and one TCGA cohort. Methylated HAND2 is even more common than other classic alterations such as TP53, KRAS PIK3CA and PTEN mutations or microsatellite instability in $\mathrm{CRC}^{15,16}$. What we find here in CRC is similar to functions of HAND2 methylation in endometrial cancer. Jones et al firstly reported HAND2 hypermethylation is common and acts as a suppressor gene in premalignant endometrial lesions and early-stage endometrial cancer ${ }^{8}$. These roles of HAND2 methylation indicates HAND2 is a potential cancer-specific driver gene in early carcinogenesis, not only a passive epigenetic event. But the underling mechanism and cellular functions of HAND2 in cancer are unknown.

HAND2, a transcriptional factor, its reconstitution leads to perturbed the activation of MAPK/ERK signaling by reduction of ERK phosphorylation in this study. MAPK/ERK signaling pathway is crucial in 
growth factor stimulation and oncogenic potential in $\mathrm{CRC}^{17}$. ERKs require phosphorylation for activation in regulating cytoplasmic and nuclear targets ${ }^{18}$. In this study, we found HAND2 reconstitution could bind to ERK and reduce its phosphorylation for ERK inactivation to inhibit tumor growth. In previous study, loss of PTEN, that are frequently mutated and suppressed in tumors, can be induced in HAND2 knock-out mice ${ }^{8}$. However, loss of PTEN expression in HAND2 reconstitution cells was shown in mRNA level but not observed in protein level, which need further confirmation.

Our data have two potential major implications. Firstly, epigenetic alterations, such as HAND2 methylation, are important functional event during early tumorigenesis and not just cancer passive characteristics. Although HAND2 are found to act crucial roles in development, mainly focusing on regulating downstream targets as one transcription factor $12,13,19$, only one recent study reveals epigenetic silencing roles of HAND2 in early carcinogenesis of endometrial cancer ${ }^{8}$, it remains unclear whether HAND2 methylation has functional relevance in other types of cancers. In our study, we firstly identify HAND2 methylation is a frequent epigenetic event in CRC and is functionally relevant to gene silencing. The downstream classic MAPK/ERK signaling of HAND2 is clarified and HAND2 acts as a tumor suppressor in CRC.

Secondly, CRC is the third most common cancer, but over $50 \%$ of CRC tumors will develop metastasis and cause poor outcomes, while early stage CRC can obtain long survive time. Current screening tools for early detection of CRC, mainly including colonoscopy and stool-based fecal immunochemical tests, are limited in many aspects, such as the invasiveness and specificity. Epigenetic cancer specific alterations, especially in driver genes, are often early molecular events in many types of cancers ${ }^{3,8}$. The potential clinical applications of HAND2 methylation are significant in early detection of CRC and in stratifying subtypes of CRC outcomes, and also are promising in developing more epigenetic targeted therapies.

Despite these notable findings, there are still some limitations in this study: 1 ) other potential targets of HAND2 such as PTEN signaling, and EGFR-RAS signaling need to be clarified; 2 ) the mechanism of HAND2 in binding to ERK and changes of downstream key molecules in ERK signaling need to be clarified in future study; 3 ) clinical application of HAND2 methylation and expression in early detection of $\mathrm{CRC}$ needed prospective trials. Thus, further studies addressing these limitations are needed to provide more evidences of the roles of epigenetic HAND2 alteration in CRC.

\section{Methods}

\section{Tissue samples and patients}

Fresh frozen tissues of colorectal cancers and paired normal control tissues were obtained from the Sixth Affiliated Hospital of Sun Yat-Sen University (SYSU) in Guangzhou, China. This study followed local ethical protocol. One pathologist reviewed all of these tumor tissues to confirm the diagnosis. All fresh tissues were stored under $-80^{\circ} \mathrm{C}$. The tumor samples and the clinical-pathological parameters were collected from a well-run electronic CRC database of this hospital. 


\section{Informed consent}

was obtained from all patients enrolled into this CRC database. TNM stage was defined according to the 6th version of American Joint Committee on Cancer (AJCC) staging Manual. Follow-up was conducted according to the guideline of National Comprehensive Cancer Network (NCCN). The overall survival (OS) was defined as the time interval from radical surgery to death. The study was approved by Human Medical Ethics Committee of SYSU.

\section{Cell culture}

Human CRC cell lines (DLD1, RKO, SW480, HCT15 and HCT8) were all cultured in RPMI-1640 or DMEM medium (Gibco, USA), supplemented with 10\% FBS and 1\% penicillin and streptomycin (Invitrogen, USA) in the humidified incubator at $37{ }^{\circ} \mathrm{C}$ with $5 \% \mathrm{CO}_{2}$. All cell lines were purchased from American Type Culture Collection (ATCC).

\section{Cell proliferation, invasion and migration}

Cell proliferation was assessed by both real-time cellular analysis (RTCA) DP device (ACEA biosciences, USA) and cell counting kit-8 (CCK-8, Dojindo lab, Japan) as our previous studies ${ }^{20,21}$. Cells are plated in 96-well plate at 6000-8000 cells per well and CCK-8 was added at appointed time and incubate for $2 \mathrm{~h}$ and the optical density was measured by multimode spectrum system (Thermo, USA) at 450nm. In RTCA assays, optical density of cells was seeded and automatically monitored every 15 min.

Cell invasion and migration were assessed by transwell assays with 8 um pore size of cell culture insert (Corning, USA) with or without Matrigel according to the manufacturer's protocols as our previous studies 20,21 . Appropriate density of cells were plated onto membrane coated with (invasion) or without (migration) Matrigel and fibronectin in the upper chamber of 24-well insert that contains serum free medium. The bottom chamber contained growth medium with $20 \%$ FBS. Cells were incubated for $48 \mathrm{~h}$ and then cells on the bottom of upper chamber insert were fixed by paraformaldehyde and stained with crystal violet, and images were captured on inverted microscope. All of above assays were repeated at least three times.

\section{DNA, RNA extraction}

Genomic DNA (gDNA) was extracted from fresh frozen tissues after thawing by DNeasy Blood and Tissue Kit (Qiagen) according to manufacturer's instructions. After extraction, gDNA was diluted into a total volume of 100ul and was quantified with a ND-100 spectrophotometer (NanoDrop technologies). Diluted DNA was bisulfited and converted using EZ DNA methylation Kit (Zymo Research) following the manufacturer's instructions.

Total RNA extraction and reverse transcription was described previously ${ }^{20,21}$. Total RNAs were extracted using TRIzol reagent (Invitrogen) according to the manufacturer's instructions. Reverse transcription was conducted with Revertra Ace PCR RT master mix with gDNA remover (Toyobo, Japan). 


\section{Real-time qPCR}

The qPCR assays were conducted by using Taqman methods according to the instructions. The designed primers and probes of target genes and iTaq Universal Probes Supermix were purchased (Applied Biosystems, USA). The qPCR running conditions were as follows: $95^{\circ} \mathrm{C}$ for $10 \mathrm{~min}$; and 40 cycles (denaturation at $95^{\circ} \mathrm{C}$ for $15 \mathrm{sec}$, annealing/extension temperature at $60^{\circ} \mathrm{C}$ for $1 \mathrm{~min}$ ). All assays were performed in duplicates, including a negative control without template.

The sequences of primers and probes are reserved by the company, and can be ordered through Applied Biosystems website with the following order numbers: HAND2 (Sc-25346, Santa Cruz; MAB8546, R\&D), EGFR (MA5-13070, Thermo Fisher), ERK 1/2 (Sc-514302, Santa Cruz), p-ERK (Sc-7383, Santa Cruz), PTEN (Sc-7974, Santa Cruz), Ras (3965, Cell Signaling). The housekeeping gene HPRT1(Santa Cruz, sc-376559) or Tubulin (Sc-73242, Santa Cruz) were applied as the endogenous reference gene. Relative gene expression was normalized to HPRT1 using the $2^{-\Delta \Delta C T}$ method. All Taqman qPCR assays were conducted by CFX96 Touch Real-Time PCR Detection System (Bio-Rad).

\section{Methylation PCR}

Quantitative MethyLight PCR (qMSP) assays using iTaq Universal SYBR Green Supermix (Bio-Rad, USA) by CFX96 Touch Real-Time PCR Detection System (Bio-Rad, USA). The qMSP assays were conducted according to our previously studies ${ }^{22,23}$. Briefly, the primers of HAND2 were firstly evaluated by end-point hemi-quantitative PCR. The PCR product was assayed by horizontal gel electrophoresis in $1.5 \%$ agarose gel and then the specificity of HAND2 methylated primers were identified. Bisulfite-converted DNA was loaded as templates for qMSP, including HAND2 primers, SYBR Green Supermix and water. $100 \%$ methylated and unmethylated EpiTect Methyl DNA standards (Qiagen) were used for positive and negative controls. The thermocycler conditions were as follows: $95^{\circ} \mathrm{C}$ for $15 \mathrm{~min}$ followed by 45 cycles of $95^{\circ} \mathrm{C}$ for $15 \mathrm{sec}$ and $60^{\circ} \mathrm{C}$ for $1 \mathrm{~min}$. Relative methylation percentage (RM\%) was calculated as ratio of $100 \times$ (methylation / control), in which methylation refers to the amount of methylated HAND2, while control refers to imput total bisulfite-converted DNA by ALUC4 MethyLight assay. All samples were run in duplicate assays. Data was analyzed by BioRad CFX manager software v3.1 and Cq was determined with Single Threshold method (Bio-Rad).

The primers sequences of HAND2 methylated site (cg02774429) were listed as follows. The probe of HAND2 is located in the +127 position of promoter region, which was indicated in previous study ${ }^{8}$.

Forward primer: CCTCTCCTTTCGAAACAAAAATCTAA;

Reverse primer: TTAGTTTAGGAGAATTATCGTCGTTATTTC.

The primer sequences of ALUC4 control, using to normalize input DNA amounts, were listed as follow:

Forward: GGTTAGGTATAGTTGTAATTTTAGTA; 


\section{SiRNA knockdown and overexpressed HAND2 plasmid}

The siRNA of HAND2 and scrambled control (si-NC) were purchased from Ribo company (Guangzhou, China). Sequences of siRNAs were persevered by the company. The siRNAs were transfected into cells by Lipofectamine RNAiMax (Invitrogen) according to instructions as our previous study.

The overexpressed HAND2 plasmid was constructed by inserting HAND2 sequence from RACE assay into pcDNA3.1+ plasmid at multiple cloning site with $\mathrm{Kpnl}$ and Xhol restriction enzymes (New England Biolabs) and was ligated using Ligation high kit (Toyobo, Japan). The constructed plasmids were transfected with cells by lipofectamine 3000 (Invitrogen) according to instructions as our previous study 21

\section{Plasmid construction and lentiviral infection}

Stable overexpression of HAND2 plasmid was constructed by ligating HAND2 to PCDH-GFP vector with in-fusion HD cloning kit (Clontech) according to manufacturer's instructions as our previous study ${ }^{21}$. PCDH-HAND2 was co-transfected with pCMV- $\triangle 8.91$ and pCMV-VSVG into 293T cells to generate viral supernatants by using lipofectamine 3000 . The lentivirus was concentrated by cold high-speed centrifuge and targeted cells were infected with lentivirus by incubating together for $48 \mathrm{~h}$. Overexpressed clones with gree lights of GFP were selected and confirmed by qRT-PCR.

\section{Western Blot}

Cells were lysed by RIPA lysis buffer (Beyotime, China) and protein was extracted and measured by BCA protein assay kit (Beibo, China). Protein were denaturated by SDS-PAGE buffer, separated by $5 \%$ stacking gel and 10\% running gel, and transferred to NC membranes (Millipore, USA). The blots were blocked with $5 \%$ non-fat milk with Tween-20 (TBST) and then incubated with primary antibody overnight and second antibody of HRP-labeled IgG for $1 \mathrm{~h}$, and scanned by enhanced chemiluminescence system (Odyssey, USA). Image $J$ software was used for quantitative analysis and proteins were normalized to internal control $\beta$-actin or Tubulin. The primary antibodies used were as follows: HAND2, GATA2, ERK, P-ERK, PTEN, RAS, Tubulin.

\section{Luciferase reporter assay}

For luciferase reporter assay, pGL4-GATA2-promoter plasmid with promoter region of GATA2 was constructed. Then the plasmid was co-transfected in CRC cell lines with pRL-TK plasmid and pcDNA3.1HAND2 overexpressed plasmid by Lipofectamine 3000. After incubating for 24-48h, luciferase activity was then measured by Dual luciferase Reporter Assay System (Promega, E1910, USA) according to manufacturer's instructions. The site mutation of pcDNA3.1-GATA2 in the promoter region site 1 (502 522bp), site 3 (471 491bp), and site 4 (1020 1040bp) were performed with KOD-PlusMutagenesis Kit (TOYOBO, SMK-101) according to manufacturer's instructions. Then co-transfection and Luciferase assays were conducted according to our previous study ${ }^{21}$. 


\section{Tumor xenograft and IHC analysis}

For construction of CRC xenograft, 5 weeks old BALB/c nude mice were purchased from laboratory animal center of SYSU (Guangzhou) and were feed in SPF conditions. HAND2 overexpressed plasmid or control plasmid was stably transfected into DLD1 cells by lentivirus method. A volume of $4 \times 10^{6}$ cells was subcutaneously injected into each BALB/c nude mice. After 24 days, all mice are euthanized by cervical dislocation and xenograft tumor were collected and weighted. Each group contained seven mice. Every 3-4 days, tumor size was recorded and calculated as volume $=\left(\right.$ length $\mathrm{X}$ width $\left.{ }^{2}\right) / 2$. Tumor tissue were fixed, wrapped and stained by anti-Ki67 according to routine $\mathrm{IHC}$ method. The details are described in our previous study ${ }^{21}$.

\section{RNA-sequencing assays}

Whole transcriptome deep sequencing (RNA-seq) was performed by Kangchen Biotech Company with Illumina Hiseq 4000 (Shanghai, China) as our previous study ${ }^{21}$. Four groups were designed, including paired DLD $1 \mathrm{siNC/siRNA}$ and overexpressed paired DLD1 pcDNA3.1/HAND2OE. Heatmap analysis was performed with OmicShare tools (a free platform for data analysis, http://www.omicshare.com/tools). Gene Ontology (GO) analysis of RNA-seq data was performed by R software. Gene Set Enrichment Analysis (GSEA) were conducted with GSEA ranked tool analysis.

\section{Chromatin immunoprecipitation (CHIP)}

For detection of HAND2 protein and GATA2 promoter DNA binding, CHIP was conducted by Chromatin IP kit (R \& D systems). Crosslink the protein-DNA complexes by incubating CRC cells with $37 \%$ formaldehyde. Then sonicate the samples to shear chromatin, centrifuge the lysates and collect supernatant. HAND2 antibody, normal IgG, or RNA polymerase II were added to the samples and incubate in ultrasonic bath. Magnetic streptavidin beads were incubated with samples and washed. The samples were boiled with a temperature-controlled water bath, and centrifuge and supernatant were collected. DNA in the sample was extracted using DNA purification kit. Purified DNA was then prepared for PCR reactions.

\section{Statistical analysis}

Student's t-tests or Chi-square test was performed appropriately for the comparisons. The correlation analyses between paired methylation and expression, correlation between expressions of different genes were performed by spearman rank correlation test. These tests were conducted using GraphPad Prism V5.01 (GraphPad Software Inc.). Potential risk factors of OS were evaluated by univariate analysis. These risk factors with $P<0.10$ in univariate analysis were included in the subsequent multivariate analysis using Cox proportional hazards model. These above tests were performed by SPSS version 17.0 software (Chicago, IL, USA). All $P$-values reported were two-sided and the difference with a $P<0.05$ was considered to be statistically significant.

\section{Conclusions}


HAND2 is one tumor suppressor by targeting ERK signaling and one potential epigenetic driver gene in CRC.

\section{Abbreviations}

CRC: colorectal cancer; HAND2: heart and neural crest derivatives expressed 2; CHIP: Chromatin immunoprecipitation; GO: Gene Ontology; OS: overall survival; qMSP: Quantitative MethyLight PCR; GSEA: Gene Set Enrichment Analysis.

\section{Declarations}

\section{Ethics approval and consent to participate}

This study was approved by Human Medical Ethics Committee of the Sixth Affiliated Hospital of Sun YatSen University (SYSU) in Guangzhou, China. Informed consent was obtained from all patients

\section{Consent for publication}

All of authors has approved the submitted manuscript and publication.

\section{Availability of data and materials}

All data generated or analysed during this study are included in this published article and its supplementary information files.

\section{Competing interests}

The authors declare no conflicts of interest.

\section{Author's Contributions}

All authors read and approved the final manuscript.

Study concept and design: Zixu Yuan, Xihu Yu, Lei Wang, William M. Grady, Hui Wang; collection of clinical specimen and data: Zixu Yuan, Xihu Yu, Chun Chen, Jian Cai, Daici Chen, Zhijie Wu, Wenle Chen; statistical analysis of data: Zixu Yuan, Xihu Yu, Zhijie Wu, Wenle Chen; manuscript writing and revision: Zixu Yuan, Xihu Yu, Chun Chen, William M. Grady, Hui Wang.

\section{Acknowledgements}

We thank Professor Lei Wang, my mentor, for the designment and support to our study. He has passed for cancer. This study is in remember of his contributions, supervise, guide and personal aid in my career.

\section{Funding}


This study was funded by National Natural Science Foundation of China (NSFC) grant (8210101440, 8157120115, 81803163), Natural Science Foundation of Guangdong PhD Start Grant (2018A030310320).

\section{References}

1. Siegel RL, Miller KD, Jemal A. Cancer statistics. 2020. CA: a cancer journal for clinicians. 2020;70(1):7-30.

2. Sharma S, Kelly TK, Jones PA. Epigenetics in cancer. Carcinogenesis. 2010;31(1):27-36.

3. De Carvalho DD, Sharma S, You JS, et al. DNA methylation screening identifies driver epigenetic events of cancer cell survival. Cancer cell. 2012;21(5):655-67.

4. Luo Y, Kaz AM, Kanngurn S, et al. NTRK3 is a potential tumor suppressor gene commonly inactivated by epigenetic mechanisms in colorectal cancer. PLoS Genet. 2013;9(7):e1003552.

5. A stool DNA. test (Cologuard) for colorectal cancer screening. Jama. 2014;312(23):2566.

6. Yuan Z, Xu T, Cai J, et al. Development and Validation of an Image-based Deep Learning Algorithm for Detection of Synchronous Peritoneal Carcinomatosis in Colorectal Cancer. Annals of surgery. 2020. doi:10.1097/SLA.0000000000004229.

7. Portela A, Esteller M. Epigenetic modifications and human disease. Nature biotechnology. 2010;28(10):1057-68.

8. Jones A, Teschendorff AE, Li Q, et al. Role of DNA methylation and epigenetic silencing of HAND2 in endometrial cancer development. PLoS Med. 2013;10(11):e1001551.

9. Luo Y, Wong CJ, Kaz AM, et al. Differences in DNA methylation signatures reveal multiple pathways of progression from adenoma to colorectal cancer. Gastroenterology. 2014;147(2):418-29.e8.

10. Kalari S, Pfeifer GP. Identification of driver and passenger DNA methylation in cancer by epigenomic analysis. Advances in genetics. 2010;70:277-308.

11. Srivastava D, Cserjesi $P$, Olson EN. A subclass of bHLH proteins required for cardiac morphogenesis. Science. 1995;270(5244):1995-9.

12. D'Autreaux F, Margolis KG, Roberts J, et al. Expression level of Hand2 affects specification of enteric neurons and gastrointestinal function in mice. Gastroenterology. 2011;141(2):576-87. 87.e1-6.

13. Li Q, Kannan A, DeMayo FJ, et al. The antiproliferative action of progesterone in uterine epithelium is mediated by Hand2. Science. 2011;331(6019):912-6.

14. Galli A, Robay D, Osterwalder M, et al. Distinct roles of Hand2 in initiating polarity and posterior Shh expression during the onset of mouse limb bud development. PLoS Genet. 2010;6(4):e1000901.

15. Barault L, Veyrie N, Jooste $\mathrm{V}$, et al. Mutations in the RAS-MAPK, $\mathrm{PI}(3) \mathrm{K}$ (phosphatidylinositol-3-OH kinase) signaling network correlate with poor survival in a population-based series of colon cancers. International journal of cancer. 2008;122(10):2255-9.

16. Yamane LS, Scapulatempo-Neto C, Alvarenga L, et al. KRAS and BRAF mutations and MSI status in precursor lesions of colorectal cancer detected by colonoscopy. Oncol Rep. 2014;32(4):1419-26. 
17. Blume-Jensen P, Hunter T. Oncogenic kinase signalling. Nature. 2001;411(6835):355-65.

18. Fang L, Lu W, Choi HH, et al. ERK2-Dependent Phosphorylation of CSN6 Is Critical in Colorectal Cancer Development. Cancer Cell. 2015;28(2):183-97.

19. Osterwalder M, Speziale D, Shoukry M, et al. HAND2 targets define a network of transcriptional regulators that compartmentalize the early limb bud mesenchyme. Dev Cell. 2014;31(3):345-57.

20. Yuan Z, Yu X, Ni B, et al. Overexpression of long non-coding RNA-CTD903 inhibits colorectal cancer invasion and migration by repressing Wnt/beta-catenin signaling and predicts favorable prognosis. Int J Oncol. 2016;48(6):2675-85.

21. Yu X, Yuan Z, Yang Z, et al. The novel long noncoding RNA u50535 promotes colorectal cancer growth and metastasis by regulating CCL20. Cell Death Dis. 2018;9(7):751.

22. Yu M, O'Leary RM, Kaz AM, et al. Methylated B3GAT2 and ZNF793 Are Potential Detection Biomarkers for Barrett's Esophagus. Cancer Epidemiol Biomarkers Prev. 2015;24(12):1890-7.

23. Luo $Y$, Tsuchiya KD, II Park $D$, et al. RET is a potential tumor suppressor gene in colorectal cancer. Oncogene. 2013;32(16):2037-47.

\section{Figures}
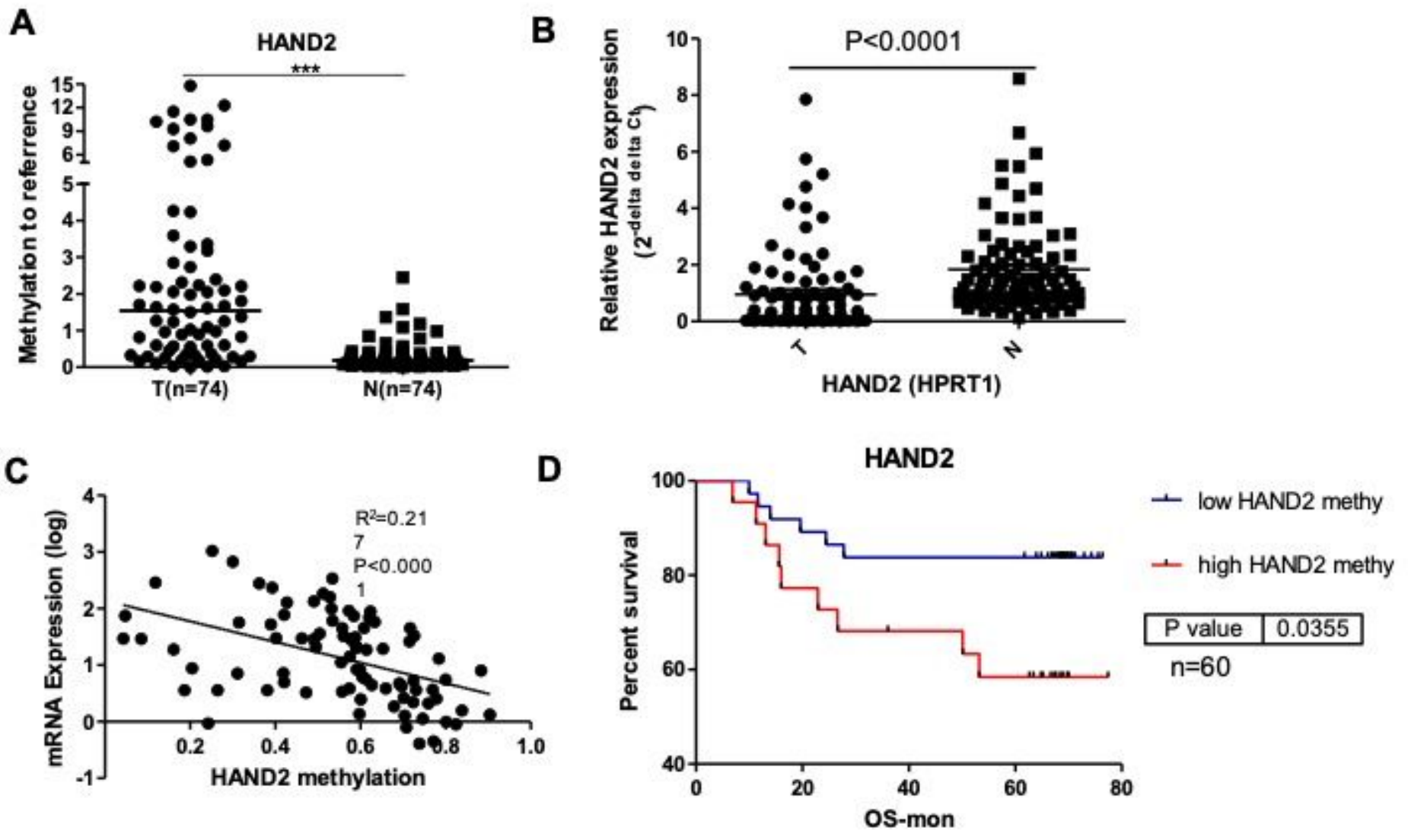

Figure 1

Figure 1 
Hypermethylated HAND2 was correlated with silenced expression and predicted poor survival. A. The methylation levels in tumor were obvious increased than paratumor normal tissue by qMSP $(n=74$ pairs, paired $t$ test, $P<0.001$, the internal control was ALUC4). B. The mRNA expression of HAND2 in tumor were reduced than paratumor normal tissue by $\mathrm{qPCP} \bigotimes n=86$ pairs $₫$ paired $t$ test $₫ \mathrm{P}<0.001 \rrbracket$ the internal control was HPRT1). C. The mRNA levels of HAND2 was negatively correlated with its methylation levels $\triangle n=61$ pairs, paired $t$ test, $r 2=0.217, P<0.0001 \rrbracket$. D. High HAND2 methylation obtained better 5 year-overall

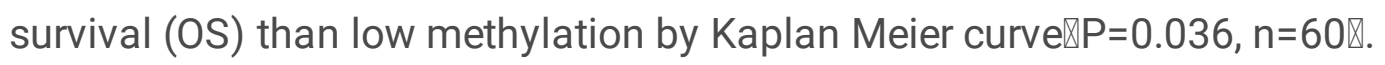

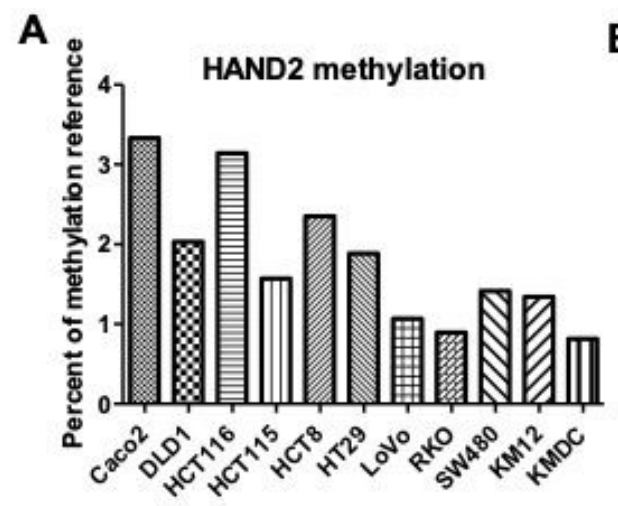

B
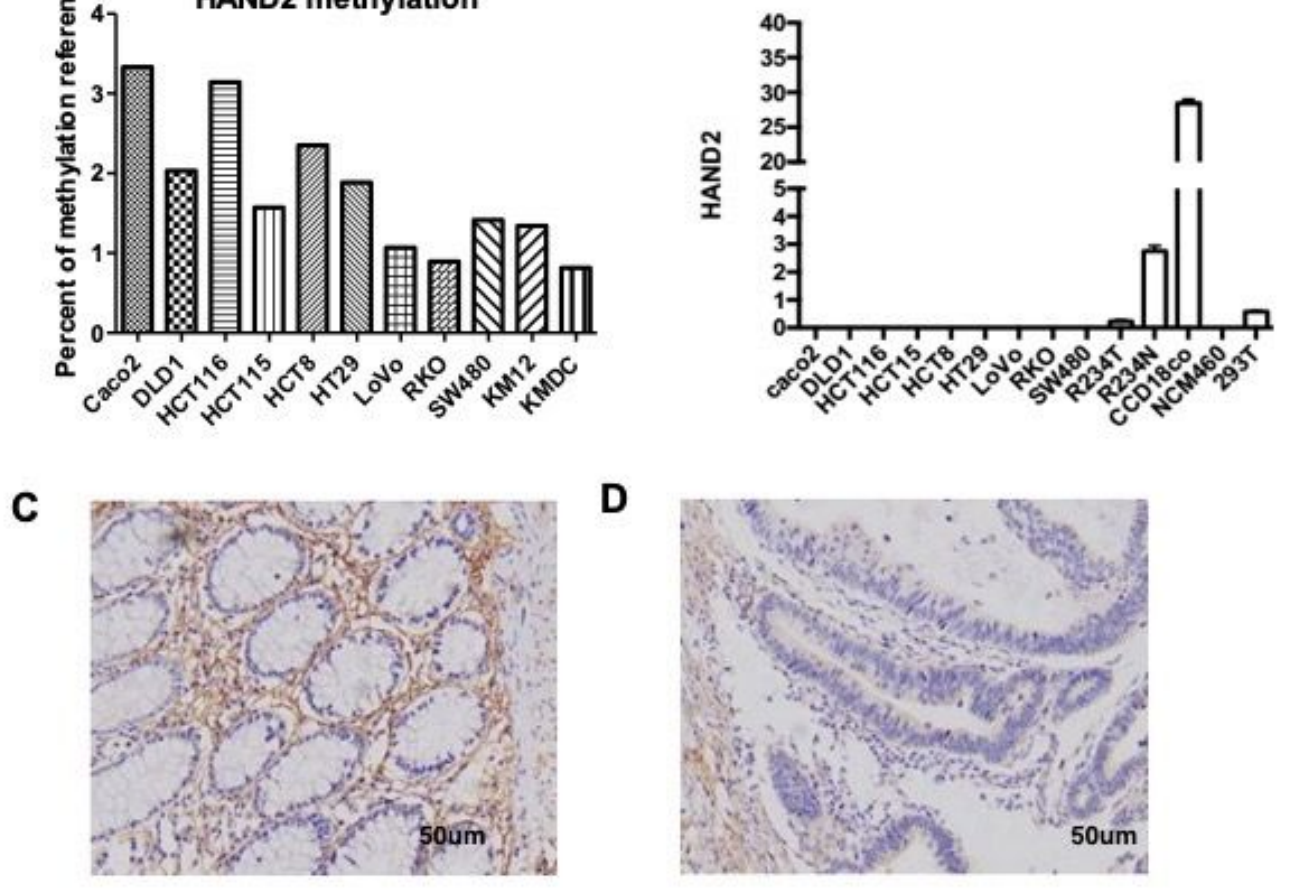

HAND2 expression in normal fissue

D

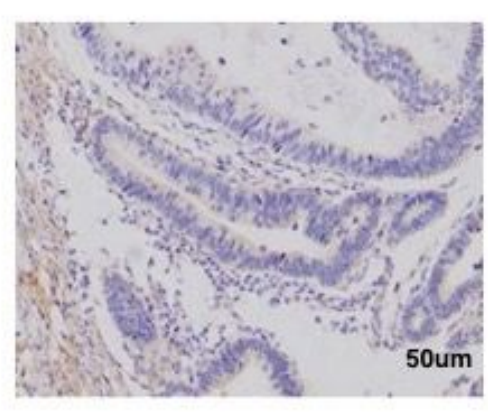

HAND2 expression in CRC tissue
HAND2-cellines

$E$

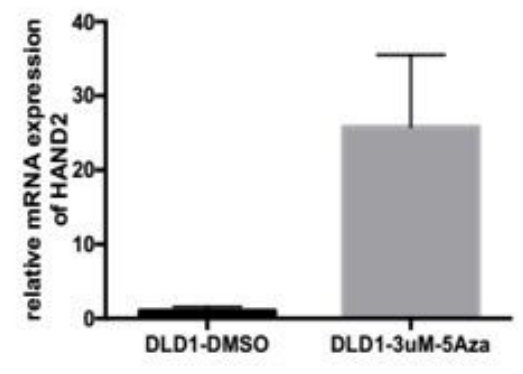

Figure 2

\section{Figure 2}

Methylated HAND2 silenced expression in CRC. A. Most of CRC cells were presented with HAND2 hypermethylation. B. HAND2 mRNA expression was greatly decreased in CRC cells compared to normal colon cell CCD18co, NCM460 or normal cell 293T. Decreased HAND2 expression in the tumor tissue (R234T) when comparing to paired normal tissue (R234N) in one CRC patient for example. C-D. The protein level of HAND2 was high in paratumor normal colon mucosa (C) while was decreased significant in tumor tissue (D) of CRC patient by IHC assay. 

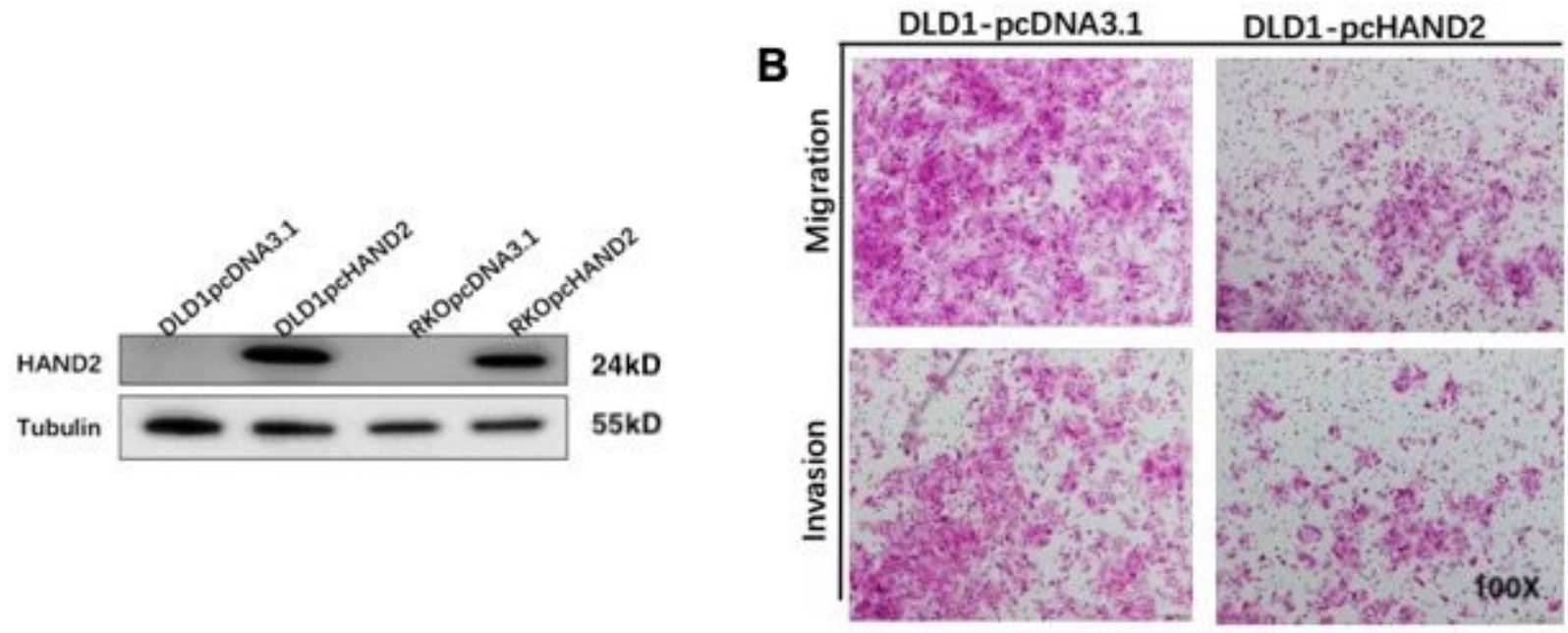

C
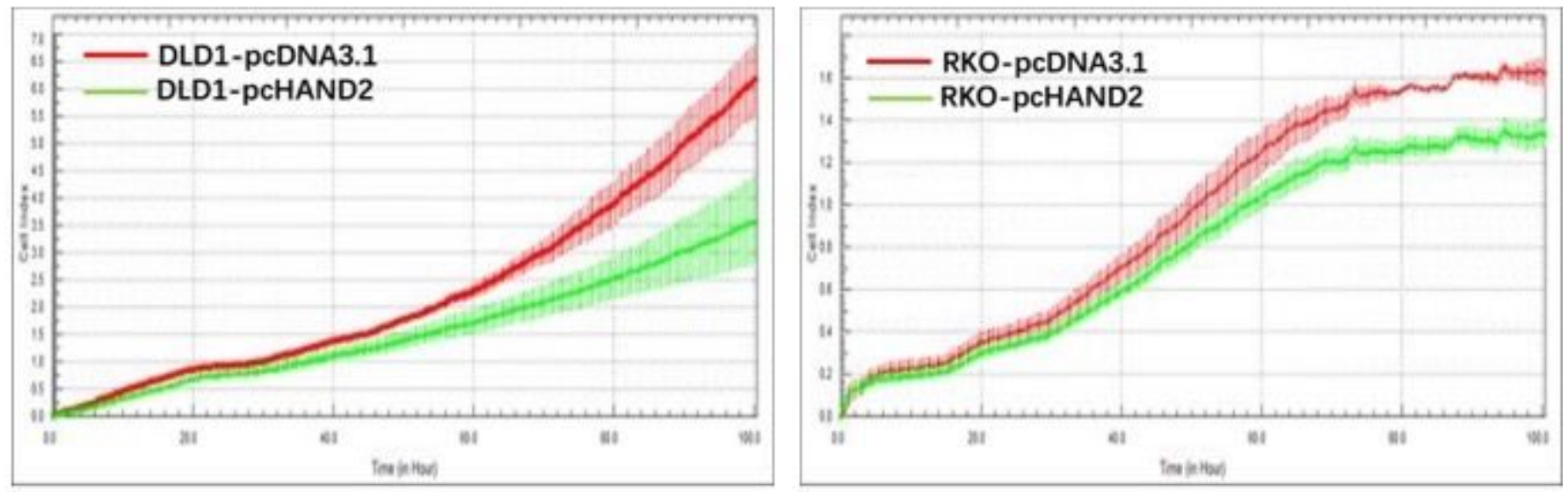

Figure 3

\section{Figure 3}

HAND2 reconstitution could repress cell proliferation, invasion and migration. A『ln DLD1 and RKO cells, HAND2 overexpressing plasmid (pcHAND2) or control vector (pcDNA3.1) were transfected with cells. Protein level of HAND2 were greatly increase after reconstitution, which revealed the successful construction of HAND2 overexpressing cells. B. The invasion and migration capacities in DLD1 cells with HAND2 reconstitution (pcHAND2) were decreased than control cells (pcDNA3.1) by 24-well transwell assays $(n=3)$. C. Cells with HAND2 reconstitution (pcHAND2) could inhibit cell proliferation than control cells (pcDNA3.1) by RTCA assay in both DLD1 and RKO cells $(n=3)$. 

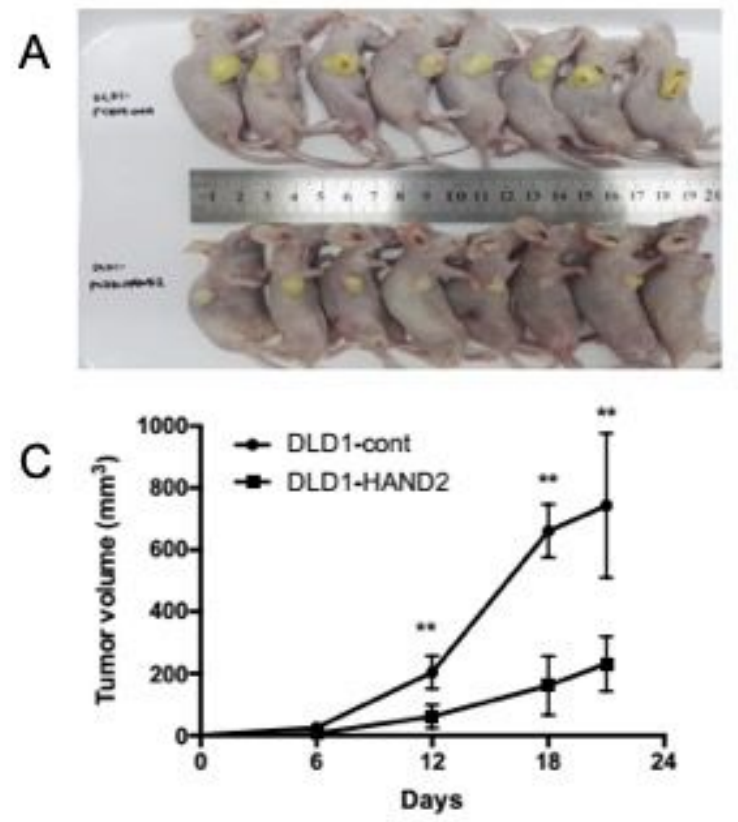
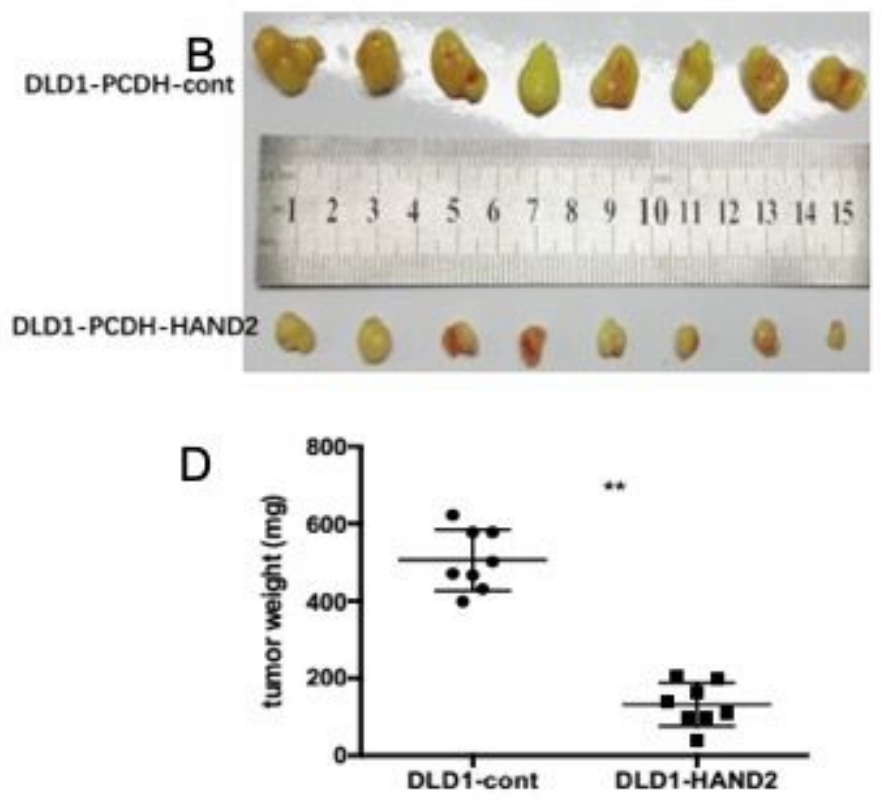

Figure 4

\section{Figure 4}

HAND2 could repress tumor growth in CRC tumor xenograft. The tumor in mice (A) and the size (B-C) and weight (D) of resected tumors were significantly decreased in mice with HAND2 reconstitution compared control mice ( $* * P<0.01$ ). The mice were injected with DLD1 cells with HAND2 reconstitution (DLD1HAND2) or control vector (DLD1-cont) and were sacrificed 12d, 18d and 24d after injection subcutaneously.
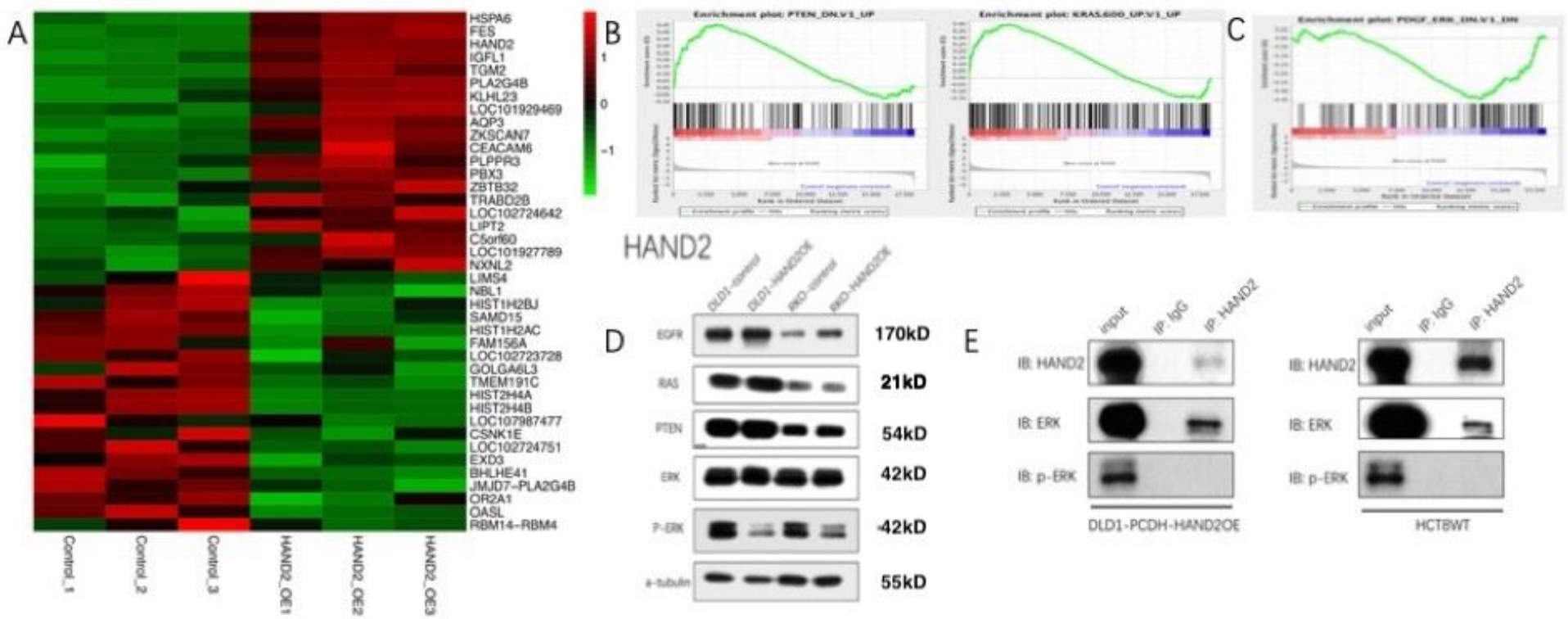

Figure 5

Figure 5 
HAND2 could regulate MAPK/ERK signaling pathway by reducing phosphorylation of ERK. A. Deep RNA sequencing were conducted to compare the mRNA expression profile between DLD1 cells with HAND2 reconstitute cells (HAND2-OE) and control vector. (Control) $(n=3)$. Thousands of aberrant expressed genes were identified in heatmap analysis. B-C. Both PTEN and KRAS pathways were upregulated (B) while ERK signaling were downregulated (C) after HAND2 reconstitution by GSEA analysis of RNA-seq data. D.

Decreased P-ERK were observed in HAND2 overexpressed cells (HAND2OE), while no significant changes of EGFR, RAS, PTEN and ERK were shown in both DLD1 and RKO cells by western blot. E. HAND2 could directly bind to ERK in both cells with HAND2 constitution (DLD1-PCDH-HAND2OE) and wild type CRC cells (HCT8WT), while the binding capacity with p-ERK was greatly decreased by CoIP assays.

\section{Supplementary Files}

This is a list of supplementary files associated with this preprint. Click to download.

- SupplementFigureS1.jpg

- SupplementFigureS2.jpg

- SupplementFigureS3.jpg

- SupplementFigureS4.jpg 